\title{
Synchrotron radiation X-Ray fluorescence elemental mapping in healthy versus malignant prostate tissues provides new insights into the glucose-stimulated zinc trafficking in the prostate as discovered by MRI.
}

Veronica Clavijo Jordan ${ }^{* 1,2}$, Alia Al-Ebraheem ${ }^{3}$, Kalotina Geraki ${ }^{4}$, Erica Dao ${ }^{5}$, Andre F. Martins $^{1,6,7}$, Sara Chirayil ${ }^{1}$, Michael Farquharson ${ }^{3}$, and A. Dean Sherry ${ }^{1,6,8}$

${ }^{1}$ Advanced Imaging Research Center and Department of Radiology, University of Texas Southwestern Medical Center, Dallas, Texas, 75390, USA.

${ }^{2}$ Athinoula A. Martinos Center for Biomedical Imaging, Massachusetts General Hospital, Harvard Medical School, Charlestown, Massachusetts, 02129 USA.

${ }^{3}$ School of Interdisciplinary Science, McMaster University, Hamilton, Ontario, L8S 4K1, Canada.

${ }^{4}$ Diamond Light Source, Harwell, Didcot OX11 0DE, U.K.

${ }^{5}$ Department of Physics and Astronomy, McMaster University, Hamilton, Ontario, L8S 4K1, Canada.

${ }^{6}$ Department of Chemistry, University of Texas at Dallas, Richardson, Texas, 75080, USA.

${ }^{7}$ Werner Siemens Imaging Center, Eberhard Karls University Tuebingen, Tuebingen, 72076, Germany.

${ }^{8}$ Vitalquan, LLC. Dallas, Texas, 75235 USA.

\section{Corresponding Author:}

* Veronica Clavijo Jordan, Ph.D.

Athinoula A. Martinos Center for Biomedical Imaging

Massachusetts General Hospital - Harvard Medical School

14913 TH St. Suite 2301

Charlestown, MA 02129

E-mail: mclavijojordan@mgh.harvard.edu

\section{Synthesis of GdL2 and GdL1}

Two versions of Gd-based zinc sensors were used in the reported studies. Synthetic procedures have been reported elsewhere and were synthesized identically here (ref. 22).

\section{In vivo MRI}

Male C57B16 mice were obtained from in-house breeding, Transgenic Adenocarcinoma of the Mouse Prostate (TRAMP) mice were obtained from Jackson Laboratories at 6-8 weeks of age. Mice (23-23 week-old) were anaesthetized with isofluorane and imaged at $4.7 \mathrm{~T}$ using a Varian/Agilent scanner, and a $12 \mathrm{~cm}$ custom-built quadrature birdcage coil. Two baseline ge $3 \mathrm{~d} \mathrm{~T}_{1^{-}}$ weighted scans were obtained $\left(\mathrm{TE} / \mathrm{TR}=1.69 / 3.34 \mathrm{~ms}\right.$, flip angle $=20^{\circ}$, NEX 8, Matrix $128 \times 128$ 
x 128, Field of view $=30 \times 30 \mathrm{~mm}$ ) and mice then received: (1) $0.07 \mathrm{mmol} / \mathrm{kg} \mathrm{GdL1}$ or GdL2 (i.v.) plus $2.2 \mathrm{mmol} / \mathrm{kg}$ glucose (i.p.) or (2) $0.07 \mathrm{mmol} / \mathrm{kg} \mathrm{GdL} 2$ plus saline as a control. Immediately after injections, sequential 3D $\mathrm{T}_{1}$-weighted scans were collected over $10 \mathrm{~min}$.

Ten TRAMP mice received $0.07 \mathrm{mmol} / \mathrm{kg}$ GdL2 (i.v.) plus $2.2 \mathrm{mmol} / \mathrm{kg}$ glucose (i.p.) and were sacrificed after 10 minutes to excise the entire prostate for Synchrotron Radiation X-Ray fluorescence analysis.

Regions of interest (ROIs) were drawn the entire prostate and avoiding the urethra. The ROIs were analyzed by calculating contrast to noise ratio (CNR). Where the signal intensity of the prostate was subtracted from the signal intensity (SI) of an ROI of back muscle and divided by the standard deviation of noise. $\mathrm{CNR}=\left(\mathrm{SI}^{\text {prostate }}-\mathrm{SI}^{\text {Muscle }}\right) / \mathrm{St}_{\mathrm{dev}}{ }^{\text {noise }}$. A change in $\mathrm{CNR}$ was then calculated by obtaining the difference between $\mathrm{CNR}^{7 \mathrm{~min}}-\mathrm{CNR}^{\text {baseline }}$.

\section{Histology and sample preparation}

The mouse lower abdomen was shaven to prevent any contamination from fur prior to dissection. The prostate was resected maintaining all lobes, urethra, and seminal vesicles intact. Once resected and rid of any adipose tissue, the prostates were slowly frozen by exposing them to liquid nitrogen-chilled isopentane. Briefly, a 100 ml beaker was filled with isopentane and submerged in liquid nitrogen for 5 minutes. The liquid nitrogen level was slightly lower than that of isopentane. Once prostate was resected, it was placed on a small weigh boat that allowed the prostate to float when directly placed on the chilled isopentane. Prostates remained on floating weigh-boat for approximately 2-5 minutes, or until fully frozen. Samples were stored in sterile polypropylene $50-\mathrm{mL}$ tubes at $-80^{\circ} \mathrm{C}$ until the day of cryosectioning and were transported on dry ice to avoid thawing. Blocks were surrounded by OCT and mounted flat, such that the area of interest was exposed for cutting. Several (two to four) 50- $\mu \mathrm{m}$-thick cryosections (Cryoultramicrotome; Leica Microsystems, Richmond Hill, ON, Canada) were obtained per sample, interleaved by $20 \mu \mathrm{m}$-thick sections for hematoxilyn and eosin (H\&E) staining. Sections for $\mathrm{XRF}$ were mounted on custom-made slides, consisting of a thin sterile film for X-ray fluorescence (XRF) (3525 Ultralene from SPEX SamplePrep, NJ,Metuchen, USA) glued to a plastic holder. The 50- $\mu \mathrm{m}$-thick-mounted sections were loaded into a freeze dryer (FreeZone 2.5; Labconco, Kansas City, MO, USA) prechilled to $50{ }^{\circ} \mathrm{C}$ and exposed to a vacuum of $0.7-1.5$ mbar for 2-4 h. The $20 \mu \mathrm{m}$-thick sections mounted on glass slides were stained with H\&E following standard histopathology protocols. Briefly, cryosections were fixed in $100 \%$ ethanol for 5-10 minutes, followed by Millipore water rinse (3 minutes), sections were submerged in Haematoxylin for 1 minute, followed by water rinse for 30 seconds. Slides were placed in Tris buffer for 30 seconds, and washed again for 1 minute. Slides were then submerged in Eosin for 1 minute, and subsequently washed for 30 seconds. The stained sections were left to dry and covered with a glass coverslip.

\section{Micro-Synchrotron Radiation $X$-Ray fluorescence ( $\mu$ SR X-RF) imaging}

Micro-synchrotron X-ray fluorescence ( $\mu$ SRXRF) was utilized at the I18 beamline at the Diamond Light Source, UK, to provide accurate high resolution quantitative elemental maps in healthy and TRAMP prostate sections from mice. The samples were fully scanned using a $50 \mu \mathrm{m} \times 50 \mu \mathrm{m}$ beam for 1 second per pixel at two different energies $(8.2$ and $11 \mathrm{keV}$ ) to obtain accurate $\mathrm{P}, \mathrm{S}, \mathrm{Zn}$, $\mathrm{Cu}, \mathrm{Fe}$, and $\mathrm{Gd}$ maps using a 4-element Vortex detector. Additional high-resolution scans were 
obtained for a smaller $300 \mu \mathrm{m}$ x $300 \mu \mathrm{m}$ region using a $5 \mu \mathrm{m}$ x $5 \mu \mathrm{m}$ beam for 2 seconds per pixel to quantify the movement of zinc pools in the prostate acinar glands. The sample position was set at $45^{\circ}$ to the incident photon beam to provide significant suppression of the scattered incident photons in the plane of the electron orbit, without affecting the measured fluorescence signal. The metals were quantified using a thin film reference material (AXO, Dresden, Germany) in conjunction with the PyMCA software package for the analysis of $\mu$ SRXRF maps which allows for the estimation of net peak areas above the continuum and also provides the means for estimating quantitative metal concentrations, in $\mu \mathrm{g} / \mathrm{g}$. In order to compare to relevant biological measurements, metal concentrations have been converted to molar (M) using the mass for the element of interest.

\section{5. $\quad \mu S R X-R F$ elemental mapping and statistical analysis}

The concentrations maps were analyzed by differentiating lobes of the mouse prostate (ventral, lateral, and dorsal) in healthy TRAMP mice. A one-way ANOVA (IBM SPSS Statistics) was performed to compare the metal concentrations categorized by the prostate lobe in 4 different groups: healthy mouse without any glucose or GdL2, healthy mouse after administration of GdL2, healthy mouse after administration of GdL2 and glucose, and TRAMP after administration of GdL2 and glucose). Fe, $\mathrm{Cu}, \mathrm{Zn}$, and, Gd concentration maps were obtained and co-registered to adjacent sectioned H\&E stained slices, and to the corresponding MRI image. The results revealed significant lower $\mathrm{Zn}$ levels in the lateral lobes of TRAMP samples compared to all others. Combining the two types of investigation, MRI and $\mu$ SRXRF, maximized the knowledge of the mechanism of glucose-stimulated zinc secretion in the prostate cancer.

\section{Glucose stimulated copper movement}

A)

\section{B)}
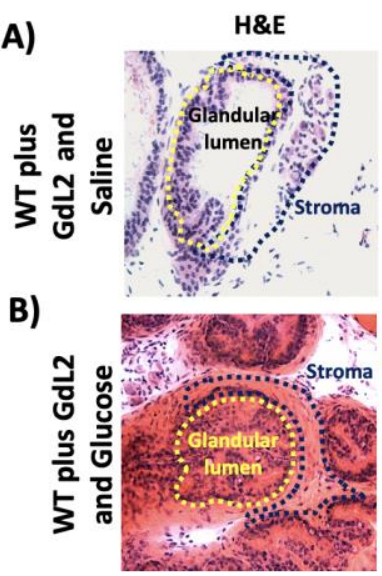

C)

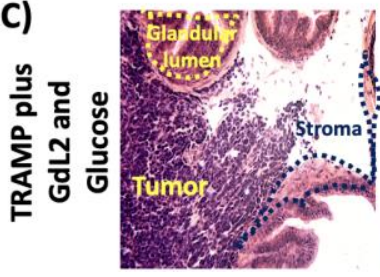

$\mathrm{Cu}$
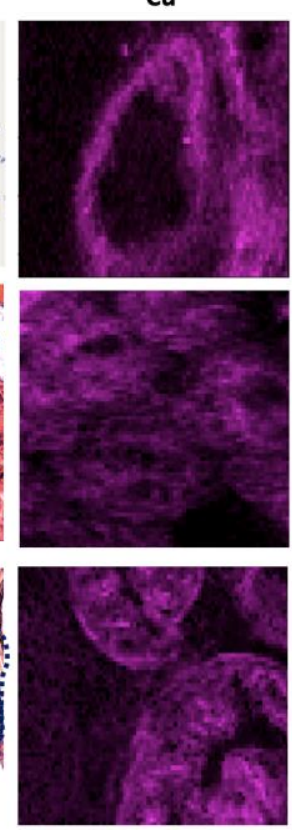

$\mathrm{Zn+Cu}$
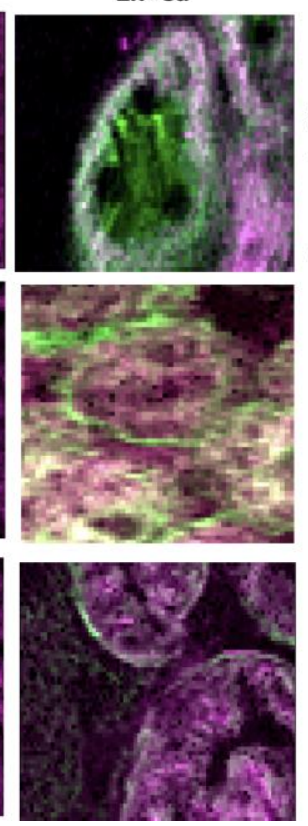

D)

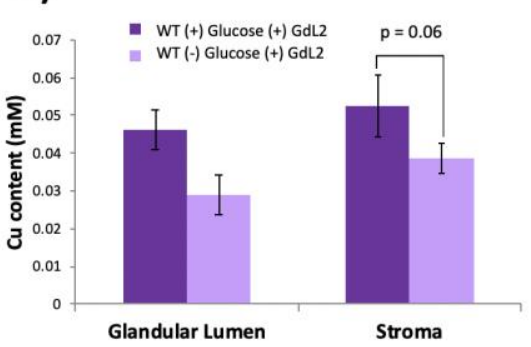


Figure S1. H\&E and $\mu$ SRXRF of a prostate gland from $\mathbf{A}$ ) healthy mouse after administration of GdL2 and saline. B) healthy mouse after receiving GdL2 and $2.2 \mathrm{mmol} / \mathrm{Kg} \mathrm{D}$-glucose IP, and C) TRAMP mouse after receiving GdL2 and $2.2 \mathrm{mmol} / \mathrm{Kg}$ D-Glucose IP. D) Quantified copper content in the glandular and stromal compartments of healthy mice receiving saline or glucose.

\section{In vivo MRI and SR XRF with high affinity for $\mathrm{Zn}$ GdL1}

A)

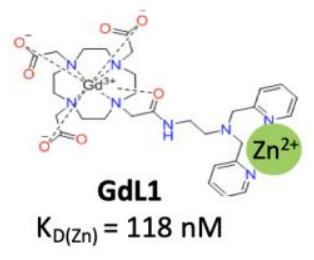

B)

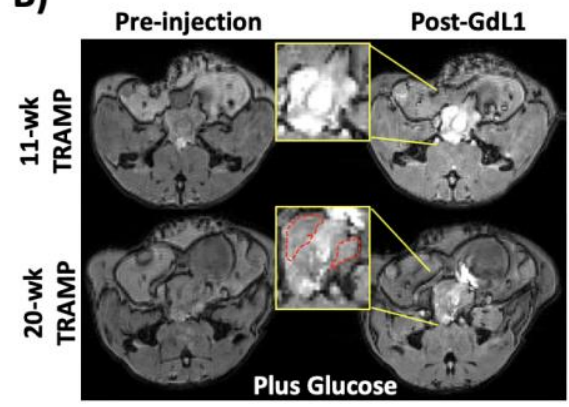

C)

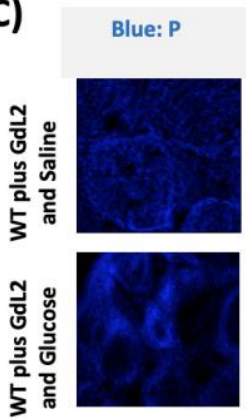

Green: Zn
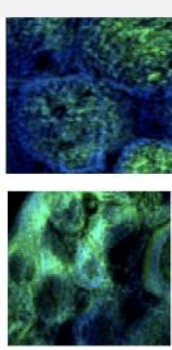

Red: S
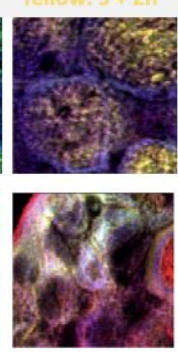

Figure S2. A) Gd-based zinc sensor with high affinity for $\mathrm{Zn}$. B) In vivo MRI of young TRAMP animal (11-weeks) and 20-wk TRAMP mouse with a large tumor found behind prostate (not visible in MRI slice). C) $\mu \mathrm{SR}$-XRF elemental maps of lateral lobe of healthy mouse showing localization of zinc, phosphorous, and sulfur. 